\title{
Proton Density MRI Increases Detection of Cervical Spinal Cord Multiple Sclerosis Lesions Compared with T2-Weighted Fast Spin-Echo
}

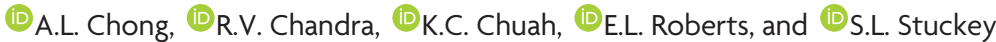

\begin{abstract}
BACKGROUND AND PURPOSE: There is a paucity of literature that supports the Consortium of Multiple Sclerosis Centers guideline that proton density MR imaging is a core spinal cord sequence. We hypothesized that proton density fast spin-echo imaging is superior to T2 fast spin-echo MR imaging for the detection of cervical cord MS lesions. This study compared the detection rate and conspicuity of cervical cord MS lesions on sagittal 1.5T proton density fast spin-echo and T2 fast spin-echo MR imaging.
\end{abstract}

MATERIALS AND METHODS: One hundred consecutive patients with MS imaged with $1.5 T$ sagittal proton density fast spin-echo and T2 fast spin-echo cervical cord MR imaging between September 2012 and October 2013 were retrospectively included. The number of MS lesions detected on each sequence was recorded; conspicuity was assessed quantitatively with the lesion-to-cord contrast ratio and lesion-contrastto-noise ratio. Statistical analysis was performed by using the Wilcoxon signed rank test.

RESULTS: Seventy-eight patients had MS cord lesions detected. Proton density fast spin-echo imaging detected a greater number of lesions ( $n=181$ ) compared with T2 fast spin-echo imaging ( $n=137, P<.001)$. Fifteen patients (19\%) with abnormal findings on proton density fast spin-echo imaging had normal findings on T2 fast spin-echo imaging; no patient with abnormal T2 fast spin-echo imaging findings had normal proton density fast spin-echo imaging findings. Although proton density fast spin-echo and $\mathrm{T} 2$ fast spin-echo imaging had similar lesion-to-cord contrast ratios (proton density fast spin-echo, $0.32 \pm 0.01$, versus T2 fast spin-echo, $0.33 \pm 0.01 ; P=.43$ ), proton density fast spin-echo had greater lesion-contrast-to-noise ratio (proton density fast spin-echo, $82 \pm 3.0$, versus T2 fast spin-echo, $64 \pm 2.6 ; P<.001$ ).

CONCLUSIONS: Proton density fast spin-echo imaging is superior to T2 fast spin-echo MR imaging for the detection of cervical cord MS lesions. Proton density fast spin-echo detects cord lesions in patients in whom T2 fast spin-echo findings appear normal. This study forms the evidentiary base for the current Consortium of Multiple Sclerosis Centers guideline that proton density imaging is a core spinal cord sequence.

ABBREVIATIONS: CMSC = Consortium of Multiple Sclerosis Centers; FSE = fast spin-echo; GRE = gradient recalled-echo; $L C C R=$ lesion-to-cord contrast ratio; $\mathrm{LCNR}=$ lesion-contrast-to-noise ratio; $\mathrm{PD}=$ proton density; $\mathrm{SE}=$ spin-echo

$\boldsymbol{S}_{\mathrm{per}}^{\mathrm{p}}$ pinal cord involvement is common in MS, particularly in the cervical cord. ${ }^{1-3}$ The detection of cord abnormality is diagnostically useful because silent cord lesions are rare in other neurologic disorders and in normal aging. ${ }^{4}$ Since the integration of MR imaging into the International Panel (McDonald) criteria in $2001,{ }^{5}$ there is increasing international effort to standardize MR

Received March 14, 2015; accepted after revision May 22.

From Monash Imaging (A.L.C., R.V.C., K.C.C., E.L.R., S.L.S.), Monash Health, Melbourne, Victoria, Australia; and Departments of Medicine (R.V.C., S.L.S.), Surgery (R.V.C.), and Imaging (S.L.S.), Monash University, Melbourne, Victoria, Australia.

Please address correspondence to Ronil V. Chandra, MBBS, MMed, FRANZCR, Monash Imaging, Monash Health, Department of Medicine and Department of Surgery, Monash University, 246 Clayton Rd, Clayton, Victoria 3168, Australia; e-mail: ronil.chandra@monashhealth.org or ronilvchandra@gmail.com

三 Indicates article with supplemental on-line table.

http://dx.doi.org/10.3174/ajnr.A4476 imaging protocols. Clinical guidelines from the Consortium of Multiple Sclerosis Centers (CMSC) recommend the use of sagittal T2-weighted and sagittal T1-weighted MR imaging and either sagittal proton density (PD) or STIR as core spinal cord sequences. ${ }^{6}$

However, a caveat of the CMSC guidelines is that the selection of spinal cord sequences was based on the experience of the consensus group, rather than large studies. ${ }^{7}$ This was not surprising due to few studies comparing pulse sequences and the disparate study designs in the literature. ${ }^{8-11}$ Perhaps the discrepancy in study designs are related to the marked variability in adherence to guideline recommendations in routine clinical practice. ${ }^{12}$ While previous studies have examined the diagnostic benefit of additional STIR imaging, ${ }^{9,13,14}$ no previous study has assessed whether there is a diagnostic benefit to the addition of sagittal PD imaging to T2-weighted imaging of the cord. Thus, the purpose of 
our study was to compare cervical cord MS lesion detection and conspicuity on sagittal 1.5T PD fast spin-echo (FSE) and T2-FSE MR imaging.

\section{MATERIALS AND METHODS Subjects}

Institutional review board approval was obtained. The requirement for informed patient consent was waived by the institutional review board. An academic teaching hospital institutional data base was retrospectively reviewed between September 2012 and October 2013. A total of 1444 patients underwent the institutional protocol for cervical cord MS lesion detection across 4 different MR imaging scanners. The study sample was formed by the first 100 consecutive patients who satisfied the following inclusion criteria: 1) cervical cord MR imaging on a single 1.5T MR scanner, 2) both sagittal PD-FSE and T2-FSE cervical cord MR imaging performed, and 3) definite MS according to the 2010 revised McDonald criteria. ${ }^{15}$ Exclusion criteria were the following: 1) MR imaging with motion artifacts reducing diagnostic quality, and 2) sagittal PD-FSE and T2FSE cervical cord MR imaging performed during separate MR imaging examinations. Thus only single MR imaging examinations were included for each patient.

\section{MR Image Acquisition}

All patients were examined on a 1.5T Magnetom Avanto (Siemens, Erlangen, Germany) MR imaging scanner equipped with an SQ-engine gradient system $(45 \mathrm{mT} / \mathrm{m}$ with slew rate of 200 $\mathrm{T} / \mathrm{m} / \mathrm{s}$ ) by using a 16-channel neck matrix coil. All patients underwent an institutional protocol for MS lesion detection consistent with the CMSC clinical guidelines: sagittal T2-FSE, sagittal T1-FSE, sagittal PD-FSE, and axial multiecho data image combination gradient recalled-echo (GRE) through the cervical cord from $\mathrm{C} 1 / 2$ to T1, with supplementary axial T2-FSE cervical cord imaging as required. No STIR imaging was performed. The Online Table summarizes the acquisition parameters of the index PD-FSE and reference T2-FSE sequences.

\section{Qualitative Lesion Detection and Analysis}

The cervical cord was divided into 7 segments (C1, C2, C3, C4, C5, C6, and C7). Two fellowship-trained neuroradiologists (R.V.C. and K.C.C. with 5 and 12 years' experience in neuroradiologic MR imaging interpretation, respectively) identified MS lesions in each segment. "MS lesions" were defined as hyperintense compared with normal-appearing cord and at least $3 \mathrm{~mm}$ in greatest dimension. ${ }^{16} \mathrm{~A}$ "long lesion" was defined as contiguous involvement of $>2$ segments. MS lesions detected on both sagittal PD-FSE and T2-FSE were included; if an MS lesion was detected on only 1 sagittal sequence, then it was included only if the same MS lesion was also detected on 1 axial sequence. This requirement minimized the inclusion of potential artifacts.

Reviewers were blinded to patient identification, clinical information, and the results of the alternate sagittal MR imaging. Blinding to image type (PD-FSE or T2-FSE) could not be performed because the imaging sequence could be easily distinguished. To maximize lesion detection, we allowed variation of window widths and levels. Recall bias was minimized by separation of each review session by 2 weeks and presentation of images
Vertebral level

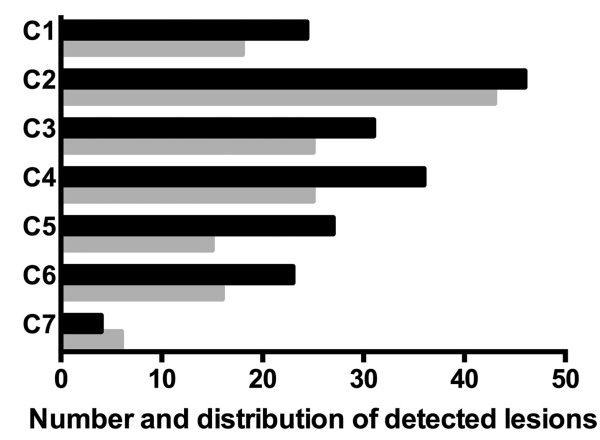

FIG 1. Comparison of the number and distribution of MS lesions detected in the cervical cord in PD-FSE (black) and T2-FSE (gray) imaging.

in a randomized order. Performance bias due to viewer fatigue was minimized by dividing the image review into 6 separate sessions. Discrepancies between the reviewers were examined in additional review sessions and were resolved by consensus. The PDFSE MR image was considered the index test, and the T2-FSE MR image was considered the reference standard, consistent with previous literature. ${ }^{8,17}$ The interobserver agreement was determined by using the $\kappa$ statistic.

\section{Quantitative Lesion Analysis}

Lesion conspicuity was assessed quantitatively by using a normalized lesion-to-cord contrast ratio (LCCR) and a lesion-contrastto-noise ratio (LCNR). ROIs were obtained within MS lesions, normal-appearing cord, and background air by using the OsiriX Imaging Software, Version 4.0 (http:// www.osirix-viewer.com). The LCCR was calculated for each sequence by applying the mean signal intensities generated in the ROIs in the equation below, where $S_{\text {lesion }}$ is the signal intensity of the lesion and $S_{\text {cord }}$ is the signal intensity of normal-appearing $\operatorname{cord}^{18}$ :

$$
\mathrm{LCCR}=\frac{S_{\text {lesion }}-S_{\text {cord }}}{S_{\text {cord }}}
$$

The LCNR was calculated for each sequence by assessing the difference between the $S_{\text {lesion }}$ and $S_{\text {cord }}$ against the level of background noise expressed as the SD of background air $\left(S D_{\text {air }}\right)$ as measured in the equation below ${ }^{18}$ :

$$
\mathrm{LCNR}=\frac{S_{\text {lesion }}-S_{\text {cord }}}{S D_{\text {air }}}
$$

The Wilcoxon signed rank test was used for statistical analysis. $P<.05$ was considered statistically significant. All statistical calculations were performed by using STATA software, Version 11 (StataCorp, College Station, Texas).

\section{RESULTS}

\section{Demographic Data}

The median age of included patients was 44.5 years (interquartile range, 37-52 years), with a female/male ratio of 3:1. No patient was excluded due to motion artifacts. The majority of patients (78/100, 78\%) had MS lesions. The mean number of lesions per patient was 2.3 (range, 0-6) for PD-FSE and 1.8 (range, 0-5) for T2-FSE imaging.

AJNR Am J Neuroradiol 37:180-84 Jan 2016 www.ajnr.org 181 

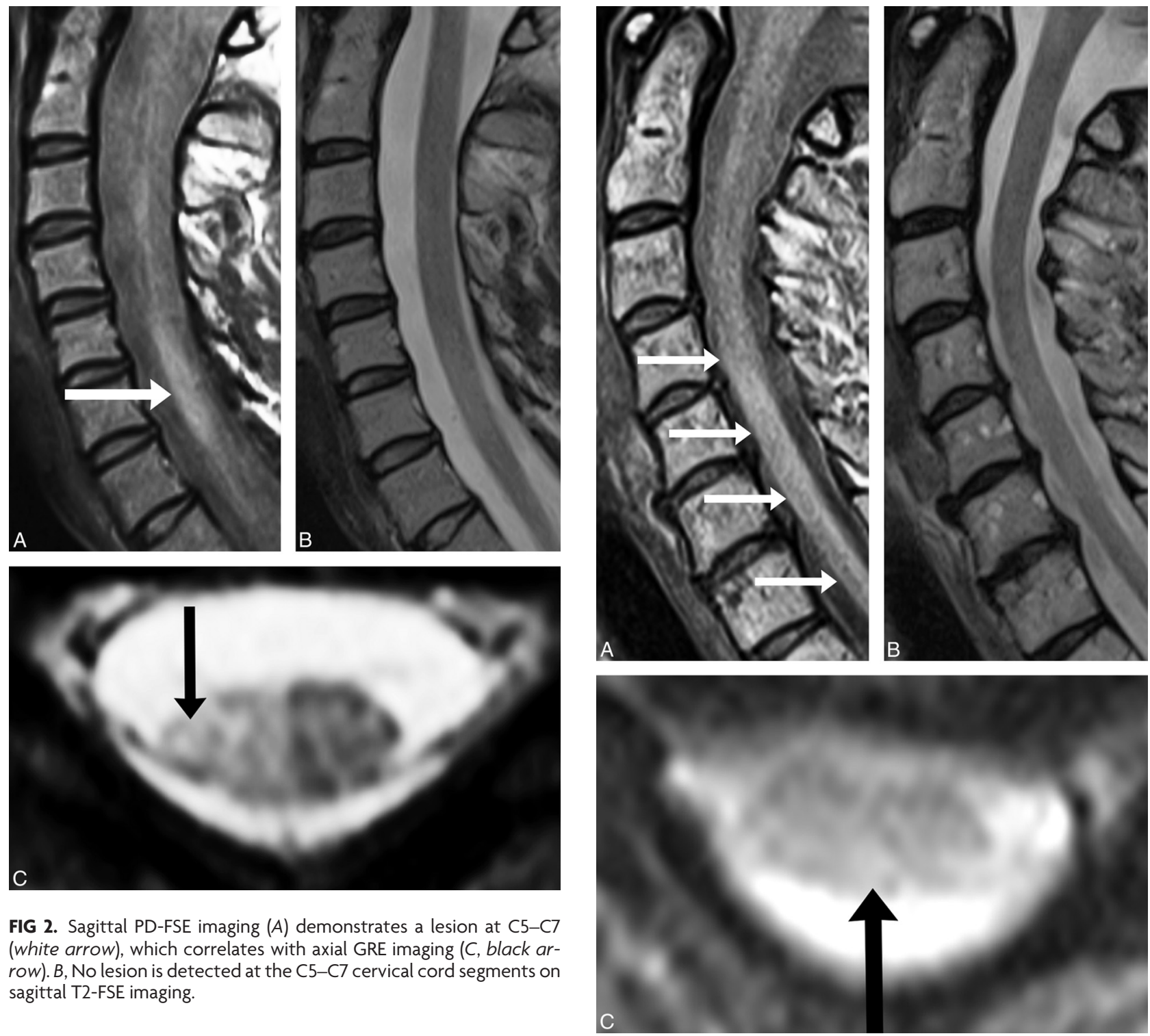

FIG 2. Sagittal PD-FSE imaging $(A)$ demonstrates a lesion at $C 5-C 7$ (white arrow), which correlates with axial GRE imaging (C, black arrow). $B$, No lesion is detected at the $\mathrm{C} 5-\mathrm{C} 7$ cervical cord segments on sagittal T2-FSE imaging.

\section{Qualitative Lesion Detection}

PD-FSE imaging depicted a greater number of lesions compared with T2-FSE imaging (PD, $n=181$, versus T2, $n=137 ; P<.001$ ). This was evident at every vertebral segment, except at the C7 level (Fig 1). There was almost perfect interobserver agreement for lesion detection on both PD-FSE $(\kappa=0.92$; 95\% CI, 0.82-1.0) and T2-FSE ( $\kappa=0.92 ; 95 \% \mathrm{CI}, 0.84-0.99)$ imaging.

Long lesions were detected in 21 patients $(21 / 78,27 \%)$ on PD-FSE imaging and 10 patients $(10 / 78,13 \%)$ on T2-FSE imaging. In 5 patients, long lesions on PD-FSE corresponded with $\geq 2$ discontinuous, discrete lesions detected on sagittal T2-FSE imaging. In all cases, axial GRE or T2 imaging also depicted contiguous involvement. In 15 patients $(15 / 78,19 \%), \geq 1$ lesion was detected on PD-FSE imaging with no lesions detected on sagittal T2-FSE imaging (Figs 2-5). All sagittal PD-FSE lesions not seen on sagittal T2-FSE imaging were confirmed on axial imaging. All PD-detected lesions were confirmed on either sagittal T2-FSE or axial imaging. One PD-detected cord lesion at $\mathrm{Cl}$ could not be confirmed on axial imaging due to lack of axial coverage and was excluded. No patients with abnormal T2-FSE imaging findings had normal PD-FSE imaging findings.

FIG 3. $\mathrm{A} C 5-\mathrm{C} 7$ lesion ( $A$, white arrows) is seen on sagittal PD-FSE imaging and correlates with axial GRE imaging (C, black arrow). C, No lesion is detected on sagittal T2-FSE imaging.

\section{Quantitative Lesion Analysis}

Although PD-FSE and T2-FSE imaging had similar LCCR (PDFSE, $0.32 \pm 0.01$, versus T2-FSE, $0.33 \pm 0.01 ; P=.43)$, PD-FSE had significantly greater LCNR (PD-FSE, $82 \pm 3.0$, versus T2FSE, $64 \pm 2.6 ; P<.001)$.

\section{DISCUSSION}

This study demonstrates that sagittal PD-FSE imaging is superior to sagittal T2-FSE MR imaging for the detection of cervical cord MS lesions at 1.5T. PD-FSE imaging detects 32\% more lesions; improved performance is evident at almost all vertebral level segments without an increase in the false-positive rate. This may be related to the greater LCNR compared with T2-FSE imaging, providing superior diagnostic confidence. The higher lesiondetection rate of PD-FSE imaging is further emphasized by the finding that PD-FSE imaging detects long lesions that are depicted as multiple smaller lesions on sagittal T2-FSE imaging. 

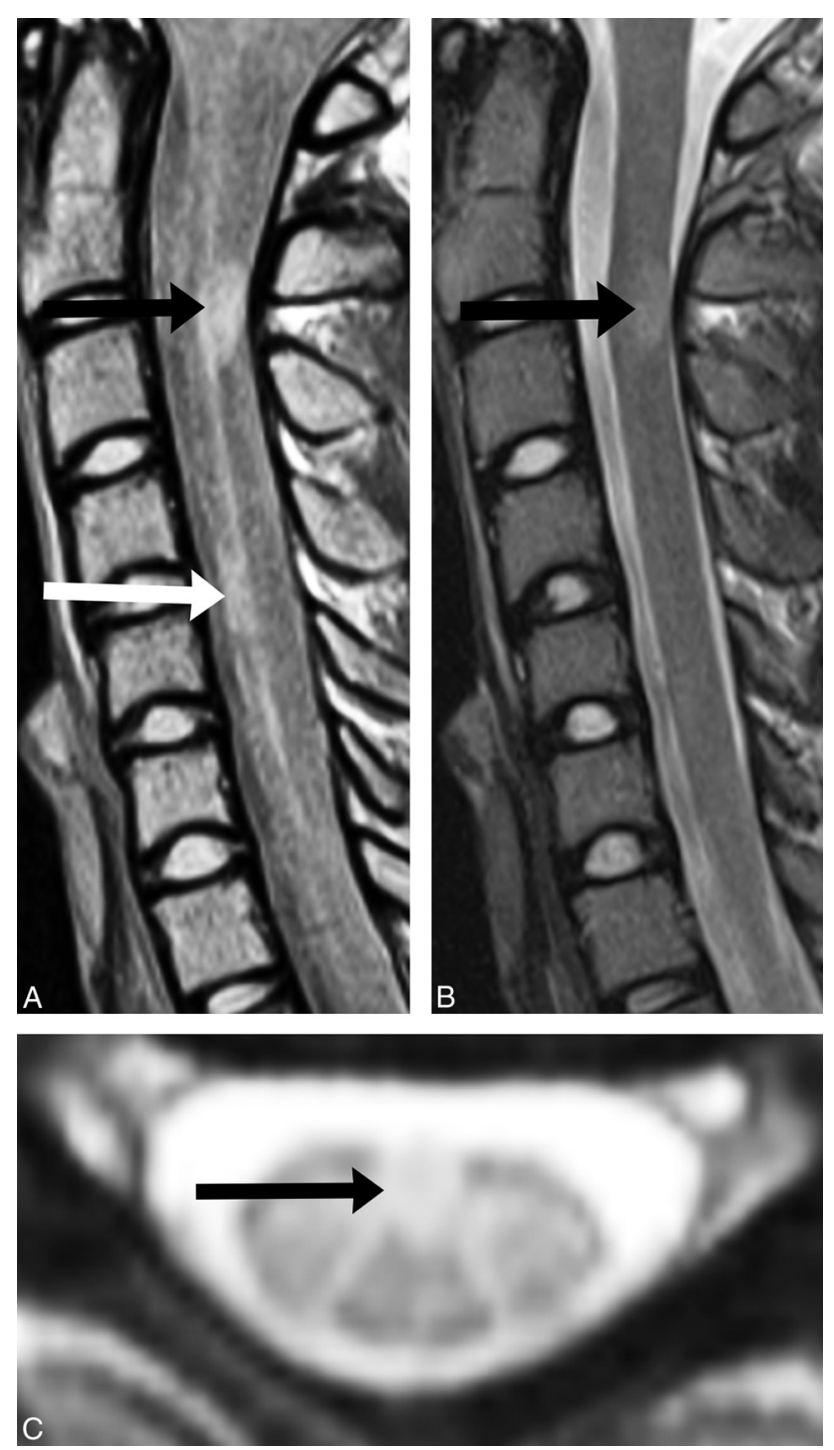

FIG 4. $A$, Sagittal PD-FSE imaging demonstrates a lesion at C2 (black arrow) and one at C4 (white arrow). B, Sagittal T2-FSE imaging demonstrates the same $\mathrm{C} 2$ lesion (black arrow), but not the $\mathrm{C} 4$ lesion. $C$, Axial GRE imaging confirms the C4 lesion (black arrow).

This result is very important since sagittal T2-FSE sequences are the most commonly used in clinical institutions for cord MS lesion detection.

In addition, almost 1 in 6 patients with definite MS had cervical cord lesions detected on PD-FSE imaging but not on T2-FSE imaging. This difference has important implications for clinical practice, as cervical cord involvement may be missed if only sagittal T2WI is performed. Most surprising, this scenario is not uncommon. A prospective case study of 14 Australian institutions by Curley et $\mathrm{al}^{12}$ found that $75 \%$ of spinal cord examinations did not comply with the CMSC MR imaging guidelines and relied on T2WI only. Specifically, PD-weighted imaging was performed in only 2 of $79(2.5 \%)$ cord examinations, and STIR imaging was performed in 18 of 79 (23\%) cord examinations (A. Coulthard, MBBS FRANZR, personal written communication, November 10, 2014).

To our knowledge, no previous studies have directly compared the lesion detection rate of PD-FSE with T2-FSE for cervical
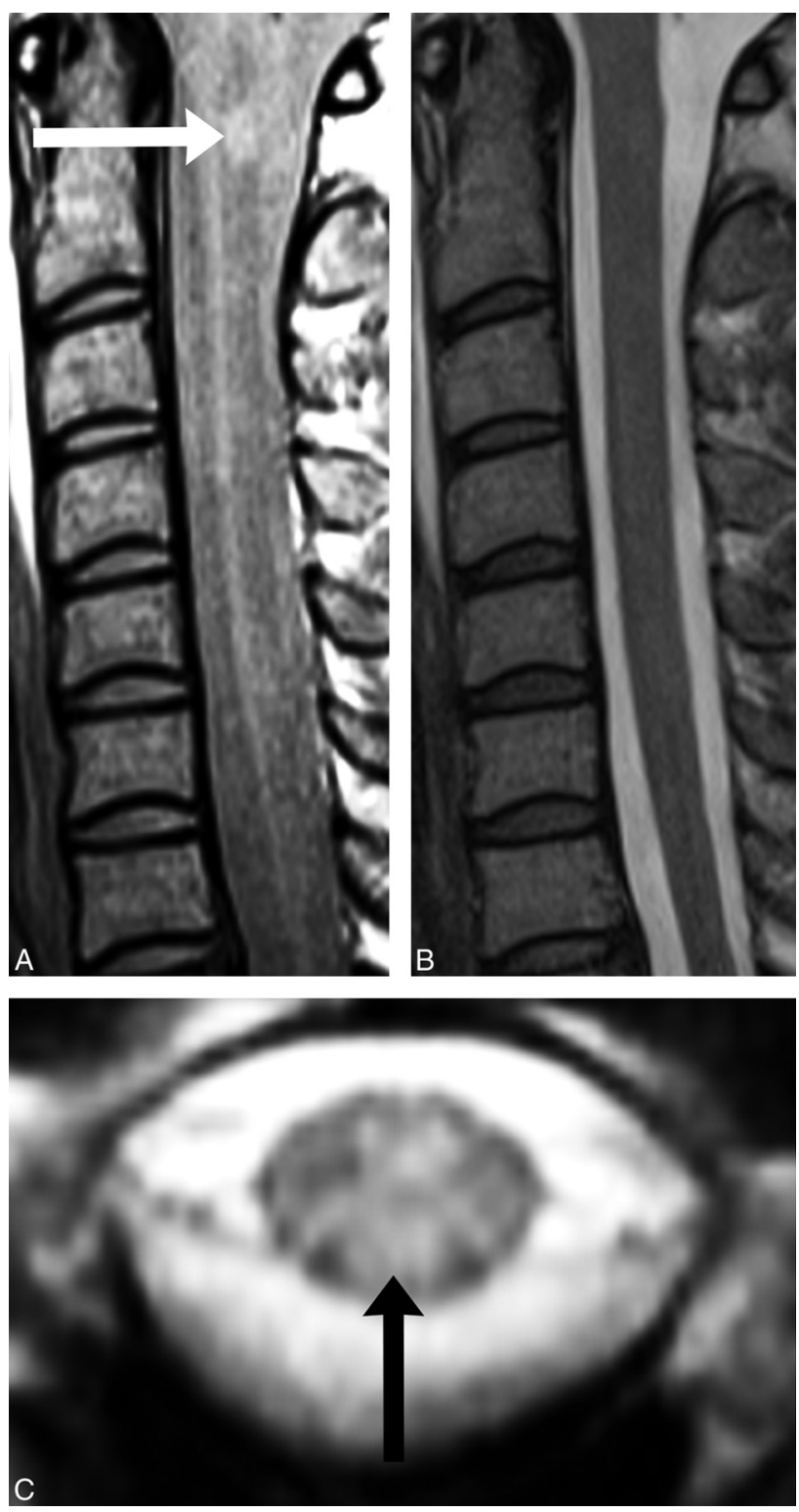

FIG 5. Sagittal PD-FSE imaging $(A)$ demonstrates a lesion at $\mathrm{Cl}$ (white arrow), which correlates with axial GRE imaging (C, black arrow). $B$, No lesion is detected at the $\mathrm{Cl}$ cord segment on sagittal T2-FSE imaging.

cord MS lesions. Most studies are designed to assess either dualecho (PD and T2-weighted) conventional spin-echo (SE) and dual-echo FSE or T2-FSE MR imaging against other novel sequences such as STIR. ${ }^{8,13,17,19}$ Discordant study designs and variability in data presentation and analyses preclude a substantive and useful comparison of our findings with those in these alternate studies. However, T2-weighted imaging was consistently outperformed by alternate MR sequences in all of these studies. ${ }^{8,13,17,19}$ Moreover, there are good histopathologic data to support the use of PD-weighted imaging. ${ }^{20}$

A postmortem study of 19 patients with MS assessed the correlation between histopathology and 4.7T and 1T PD-SE MR imaging. All areas of the spinal cord scored as abnormal by the neuropathologist were rated as abnormal on PD-SE MR imaging; all abnormal specimens were identified by both $4.7 \mathrm{~T}$ and $1 T$ PD-SE MR imaging. In addition, no abnormalities were 
detected in the 3 control patients on either histopathology or PD-SE MR imaging. ${ }^{20}$

The significantly improved LCNR in our large cohort has not been previously reported. In a smaller prospective study of 20 patients with MS, a higher contrast-to-noise ratio with PD conventional SE compared with T2 conventional SE imaging was reported, but the result did not reach statistical significance. ${ }^{10}$ Another study of 60 patients with MS measured the contrast-to-noise ratio by using $1 \mathrm{~T}$ PD SE and T2-SE imaging. Although statistical comparison was not performed, reported contrast-to-noise ratio values were approximately twice as high for PD-SE imaging as for T2-SE imaging. ${ }^{11}$ Conversely, an alternate smaller study found that T2 conventional SE had a greater contrast-to-noise ratio than PD conventional SE imaging at 1.5T in 20 patients with MS. ${ }^{9}$ This difference may simply reflect our larger cohort and imaging results on modern 1.5T MR imaging scanners compared with this previous study, which recruited almost 20 years ago.

Our study confirms the diagnostic benefit of PD-FSE MR imaging in addition to T2-FSE cervical cord imaging, as recommended by the CMSC guidelines. PD-FSE is easy to implement; our PD sequence was performed in $<3$ minutes and was welltolerated by patients. The strengths of our study include the large cohort of patients with definite MS, minimization of recall and performance bias, and the use of both qualitative and quantitative analyses. The limitations of our study are the retrospective study design and use of 1.5T MR imaging, which was chosen to increase the generalizability of the study results to smaller institutions and clinical practices without 3T MR imaging availability. Although 3T MR imaging improves the PD lesion volume detection rate in the brain compared with $1.5 \mathrm{~T},{ }^{21}$ no such data are yet available for the spinal cord. In addition, STIR sequences were not performed and/or examined in this cohort. This is our institutional practice, which is in line with the CMSC guidelines, in which sagittal PD or STIR may be performed as the core spinal cord sequences.

\section{CONCLUSIONS}

Sagittal PD-FSE imaging is superior to T2-FSE MR imaging for the detection of cervical cord MS lesions. PD-FSE detects cord lesions in patients in whom sagittal T2-FSE imaging appears normal. This study forms the evidentiary base for the current CMSC guideline that PD imaging is a core spinal cord imaging sequence.

\section{ACKNOWLEDGMENTS}

We thank Eldho Paul, School of Public Health, Monash University, for his assistance with statistical analysis.

\section{REFERENCES}

1. Tartaglino LM, Friedman DP, Flanders AE, et al. Multiple sclerosis in the spinal cord: MR appearance and correlation with clinical parameters. Radiology 1995;195:725-32 CrossRef Medline

2. Kidd D, Thorpe JW, Thompson AJ, et al. Spinal cord MRI using multiarray coils and fast spin echo, II: findings in multiple sclerosis. Neurology 1993;43:2632-37 CrossRef Medline

3. Uldry PA, Regli F, Uské A. Magnetic resonance imaging in patients with multiple sclerosis and spinal cord involvement: 28 cases. J Neurol 1993;240:41-45 CrossRef Medline
4. Bot JC, Barkhof F, Lycklama à Nijeholt G, et al. Differentiation of multiple sclerosis from other inflammatory disorders and cerebrovascular disease: value of spinal MR imaging. Radiology 2002;223: 46-56 CrossRef Medline

5. McDonald WI, Compston A, Edan G, et al. Recommended diagnostic criteria for multiple sclerosis: guidelines from the International Panel on the Diagnosis of Multiple Sclerosis. Ann Neurol 2001;50: 121-27 CrossRef Medline

6. Consortium of Multiple Sclerosis Centers. MRI Protocol for the Diagnosis and Follow-up of MS 2009 Revised Guidelines. November 10, 2012. http://c.ymcdn.com/sites/www.mscare.org/resource/collection/ 9C5F19B9-3489-48B0-A54B-623A1ECEE07B/mriprotocol2009.pdf. Accessed March 31, 2013

7. Simon JH, Li D, Traboulsee A, et al. Standardized MR imaging protocol for multiple sclerosis: Consortium of MS Centers Consensus Guidelines. AJNR Am J Neuroradiol 2006;27:455-61 Medline

8. Philpott C, Brotchie P. Comparison of MRI sequences for evaluation of multiple sclerosis of the cervical spinal cord at $3 \mathrm{~T}$. Eur J Radiol 2011;80:780-85 CrossRef Medline

9. Hittmair K, Mallek R, Prayer D, et al. Spinal cord lesions in patients with multiple sclerosis: comparison of MR pulse sequences. AJNR Am J Neuroradiol 1996;17:1555-65 Medline

10. Bot JC, Barkhof F, Lycklama à Nijeholt GJ, et al. Comparison of a conventional cardiac-triggered dual spin-echo and a fast STIR sequence in detection of spinal cord lesions in multiple sclerosis. Eur Radiol 2000; 10:753-58 CrossRef Medline

11. Lycklama à Nijeholt GJ, Barkhof F, Scheltens $P$, et al. MR of the spinal cord in multiple sclerosis: relation to clinical subtype and disability. AJNR Am J Neuroradiol 1997;18:1041-48 Medline

12. Curley M, Josey L, Lucas R, et al. Adherence to MRI protocol consensus guidelines in multiple sclerosis: an Australian multi-centre study. J Med Imaging Radiat Oncol 2012;56:594-98 CrossRef Medline

13. Rocca MA, Mastronardo G, Horsfield MA, et al. Comparison of three MR sequences for the detection of cervical cord lesions in patients with multiple sclerosis. AJNR Am J Neuroradiol 1999;20:1710-16 Medline

14. Campi A, Pontesilli S, Gerevini S, et al. Comparison of MRI pulse sequences for investigation of lesions of the cervical spinal cord. Neuroradiology 2000;42:669-75 CrossRef Medline

15. Polman CH, Reingold SC, Banwell B, et al. Diagnostic criteria for multiple sclerosis: 2010 revisions to the McDonald criteria. Ann Neurol 2011;69:292-302 CrossRef Medline

16. Geurts JJ, Roosendaal SD, Calabrese M, et al; MAGNIMS Study Group. Consensus recommendations for MS cortical lesion scoring using double inversion recovery MRI. Neurology 2011; 76:418-24 CrossRef Medline

17. Dietemann JL, Thibaut-Menard A, Warter JM, et al. MRI in multiple sclerosis of the spinal cord: evaluation of fast short-tau inversion-recovery and spin-echo sequences. Neuroradiology 2000;42:810-13 CrossRef Medline

18. Geurts JJ, Pouwels PJ, Uitdehaag BM, et al. Intracortical lesions in multiple sclerosis: improved detection with 3D double inversion-recovery MR imaging. Radiology 2005;236:254-60 CrossRef Medline

19. Poonawalla AH, Hou P, Nelson FA, et al. Cervical spinal cord lesions in multiple sclerosis: T1-weighted inversion-recovery MR imaging with phase-sensitive reconstruction. Radiology 2008;246:258-64 CrossRef Medline

20. Nijeholt GJ, Bergers E, Kamphorst W, et al. Post-mortem highresolution MRI of the spinal cord in multiple sclerosis: a correlative study with conventional MRI, histopathology and clinical phenotype. Brain 2001;124:154-66 CrossRef Medline

21. Sicotte NL, Voskuhl RR, Bouvier S, et al. Comparison of multiple sclerosis lesions at $\mathbf{1 . 5}$ and 3.0 Tesla. Invest Radiol 2003;38:423-27 CrossRef Medline 